\title{
Using Google Trends to investigate global COPD awareness
}

To the Editor:

We read the recent article published in the European Respiratory Journal by Bовнм et al. [1] with great interest: their study is the first to explore global chronic obstructive pulmonary disease (COPD) awareness using Google searches. Currently, with the development of the internet and search engines, Google Trends has emerged as a robust tool to investigate the interest of the general population in medical conditions [24]. In the study by Вовнм et al. [1], it was found that the awareness of COPD in the real world is rising, while it is highly under-represented in the public interest compared with other common conditions. In addition, public interest in seeking COPD information through Google searches presented a seasonal pattern, with peaks in the first and the fourth quarter of the year. These findings may help to improve programmes to guide interventions for COPD and contribute to the development of preventative healthcare for this disease.

Overall, the rationale, research design and statistical analysis of this study was clearly defined and appropriate, and the conclusions are supported by the results. Nevertheless, it would be more interesting to incorporate some key points in this study. First, the interest by region option was set to worldwide, thus, lacking a trend analysis of relative search volume of specific countries in the northern and southern hemisphere, respectively. Second, the survey did not state which category the assessment of search interest was based on (i.e. "all categories" or "health" categories), which may not be strict enough. Third, the findings of the study would be more comprehensive if it included the analysis of both "hot topics" and "top rising topics" [5]. The "hot topics" are the most popular topics or terms, while the "top rising topics" are terms with the largest increase in search frequency since the last period of time. In particular, analysis of "top rising topics" can give guidance for medical staff to provide their patients with valuable information.

Taken together, this Google Trends-based investigation provided new evidence for public interest and seasonal patterns in COPD. The findings of this study may serve as the basis for future studies to explore the association between Internet searches and actual clinical trends. However, more comprehensive analysis on COPD-related topics, including "hot topics" and "top rising topics", and trend analyses of the northern and southern hemispheres, are needed to derive a more precise evaluation of global COPD awareness.

Chan-Na Zhao ${ }^{1,2}$, Qian $\mathrm{Wu}^{1,2}$ and Hai-Feng Pan ${ }^{1,2}$

${ }^{1}$ Dept of Epidemiology and Biostatistics, School of Public Health, Anhui Medical University, Hefei, China. ${ }^{2}$ Anhui Province Key Laboratory of Major Autoimmune Diseases, Hefei, China.

Correspondence: Hai-Feng Pan, Dept of Epidemiology and Biostatistics, School of Public Health, Anhui Medical University, 81 Meishan Road, Hefei, Anhui, 230032, PR China. E-mail: panhaifeng1982@sina.com

Received: May 312019 | Accepted after revision: June 062019

Conflict of interest: None declared.

@ERSpublications

Google Trends provides new evidence for public interest and seasonal patterns in COPD http://bit.ly/2xsGbIE

Cite this article as: Zhao C-N, Wu Q, Pan H-F. Using Google Trends to investigate global COPD awareness. Eur Respir J 2019; 54: 1901076 [https://doi.org/10.1183/13993003.01076-2019].

1 Boehm A, Pizzini A, Sonnweber T, et al. Assessing global COPD awareness with Google Trends. Eur Respir J 2019; 53: 1900351. 
2 Jellison SS, Bibens M, Checketts J, et al. Using Google Trends to assess global public interest in osteoarthritis. Rheumatol Int 2018; 38: 2133-2136.

3 Kardes S, Kardes E. Seasonality of bruxism: evidence from Google Trends. Sleep Breath 2019; 23: 695-701.

4 Chai Y, Luo H, Zhang Q, et al. Developing an early warning system of suicide using Google Trends and media reporting. J Affect Disord 2019; 255: 41-49.

5 Wu GC, Tao SS, Zhao CN, et al. Leveraging Google Trends to investigate the global public interest in rheumatoid arthritis. Rheumatol Int 2019; 39: 1439-1444.

Copyright @ERS 2019

From the authors:

We appreciate the thoughtful comments of $\mathrm{C}-\mathrm{N}$. Zhao and co-workers regarding our research article "Assessing global COPD awareness with Google Trends" [1].

\section{CrossMark}

We kindly would like to point out that we did not observe a rise in chronic obstructive pulmonary disease (COPD)-related awareness. In fact, our study stresses the discrepancy between rising numbers in prevalence and mortality related to COPD in the real world and steady relative search volumes (RSV) in Google Trends as a surrogate parameter for COPD awareness. RSV peaks were observed during winter, as well as in the COPD awareness month, November. However, after statistically correcting for a seasonal component, no overall increase in RSV for COPD was observed.

Google Trends offers many possibilities to analyse specific search terms/topics. Notably, the ability to characterise queries according to their geographic distribution may add great scientific value to specific research questions, as shown by GINSBERG et al. [2]. Recent studies reported that search terms with seasonal

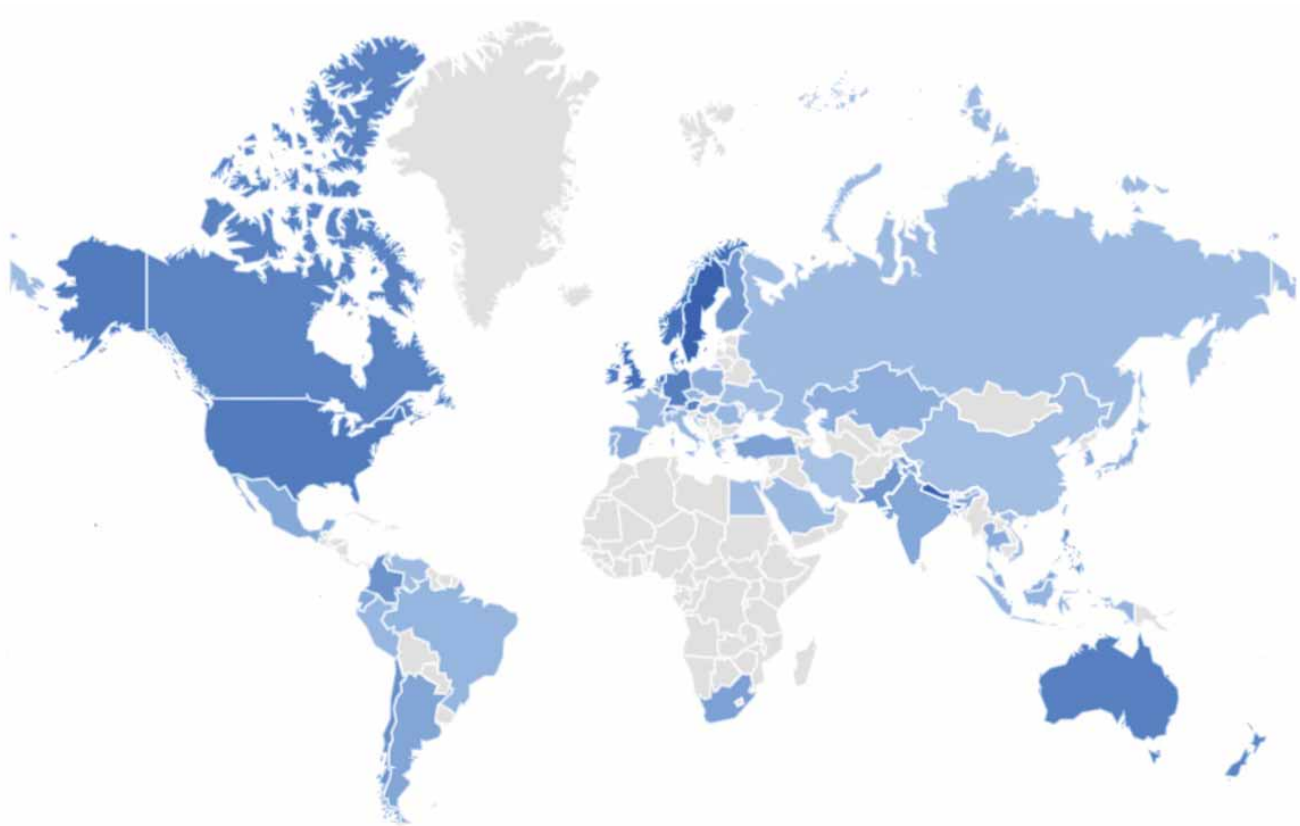

FIGURE 1 Interest by region for the Google Trends topic "chronic obstructive pulmonary disease" from 2004 to present. The figure highlights that countries in the southern hemisphere are highly under-represented (colour intensity outlines search interest). Data source: Google Trends (https://www.google.com/trends).

@ERSpublications

Google Trends provides many features that might help to identify geographic regions with low disease awareness and could contribute to a better understanding of the current needs of COPD patients http://bit.ly/2YXfGHz

Cite this article as: Boehm A, Pizzini A, Sonnweber T, et al. Using Google Trends to investigate global COPD awareness. Eur Respir J 2019; 54: 1901339 [https://doi.org/10.1183/13993003.01339-2019]. 
components may have an inverse relationship when data is split according to Northern and Southern hemisphere [3-5]. Accordingly, a study by Jenkins et al. [6] described this phenomenon when analysing seasonal trends of acute COPD exacerbations. However, when analysing Internet search queries, it is important to consider that the Northern hemisphere is dominated by high-income countries with broad Internet access and use, likely causing a bias related to the amount of Internet traffic (figure 1). Thus, we decided to compare our results with mortality data from high-income countries only. We acknowledge that our study does not assess awareness in low-income countries, where COPD is a largely neglected disease. In this context, Google Trends may help to identify geographic regions with urgent needs to improve COPD-related healthcare.

Google Trends also has the option to filter the results according to specific categories, such as "Arts \& Entertainment", "Books \& Literature", "Science" or "HEALTH". The algorithms used by Google Trends for categorisation, however, are not transparent and might change over time. We, therefore, chose the "all categories" option, which moreover will also facilitate reproducibility, an important issue when trying to establish a standardised methodology for further Google Trends-based studies.

Finally, Google Trends suggests "hot topics" and "top rising topics" (i.e. "is COPD contagious", "how do you get COPD" or "COPD life expectancy") which are powerful tools to identify matters of particular interest related to "COPD" queries. The suggested topics might help to understand the current needs of COPD patients, as they summarise the present most substantial search queries. An in-depth analysis of these topics may help to optimise patient education and public awareness, and future studies focusing on such a patient-oriented approach are heavily awaited.

Anna Boehm ${ }^{1,3}$, Alex Pizzini $\circledast^{1,3}$, Thomas Sonnweber ${ }^{1}$, Judith Loeffler-Ragg ${ }^{1}$, Claudia Lamina ${ }^{2}$, Guenter Weiss ${ }^{1}$ and Ivan Tancevski ${ }^{1}$

${ }^{1}$ Dept of Internal Medicine II, Infectious Diseases, Pneumology, Rheumatology, Innsbruck Medical University, Innsbruck, Austria. ${ }^{2}$ Division of Genetic Epidemiology, Innsbruck Medical University, Innsbruck, Austria. ${ }^{3}$ A. Boehm and A. Pizzini contributed equally to this manuscript.

Correspondence: Alex Pizzini, Dept of Internal Medicine II, Infectious Diseases, Pneumology, Rheumatology, Innsbruck Medical University, Anichstraße 35, 6020 Innsbruck, Austria. E-mail: alex.pizzini@i-med.ac.at

Received: July 062019 | Accepted: July 092019

Conflict of interest: None declared.

\section{References}

1 Boehm A, Pizzini A, Sonnweber T, et al. Assessing global COPD awareness with Google Trends. Eur Respir J 2019; 53: 1900351.

2 Ginsberg J, Mohebbi MH, Patel RS, et al. Detecting influenza epidemics using search engine query data. Nature 2009; 457: 1012-1014.

3 Platek AE, Sierdzinski J, Krzowski B, et al. Seasonal trends in hypertension in Poland: evidence from Google search engine query data. Kardiol Pol 2018; 76: 637-641.

4 Zhang X, Dang S, Ji F, et al. Seasonality of cellulitis: evidence from Google Trends. Infect Drug Resist 2018; 11: 689-693.

5 Ilias I, Alexiou M, Meristoudis G. Is there seasonality in hypothyroidism? a Google Trends pilot study. Cureus 2019; 11: e3965.

6 Jenkins CR, Celli B, Anderson JA, et al. Seasonality and determinants of moderate and severe COPD exacerbations in the TORCH study. Eur Respir J 2012; 39: 38-45. 